\title{
Abastecimiento de sangre durante desastres: la experiencia de Chile en 2010
}

\author{
Marcela Vásquez, ${ }^{1}$ Mónica Maldonado, ${ }^{1}$ Federico Tagle, ${ }^{2}$ \\ Sergio León, ${ }^{2}$ Alejandra Soto, ${ }^{2}$ Aaron Mena ${ }^{2}$ y Carla Toro $^{1}$
}

Forma de citar Vásquez M, Maldonado M, Tagle F, León S, Soto A, Mena A, et al. Abastecimiento de sangre durante desastres: la experiencia de Chile en 2010. Rev Panam Salud Publica. 2011;29(5):365-70.

RESUMEN Se realizó una revisión de la gestión del abastecimiento y suministro de sangre durante desastres a partir de las experiencias de diversos eventos ocurridos principalmente en la primera década de este siglo, y en particular el terremoto grado 8,8 en la escala de Richter que afectó la zona centro sur de Chile el 27 de febrero de 2010. El objetivo fue proporcionar información que pueda ser útil para mejorar las estrategias y planes de respuesta durante potenciales desastres futuros.

La información descriptiva sobre los procedimientos de respuesta se obtuvo mediante entrevistas, reportes internos y la base de datos del sistema informático del Centro Productivo Regional de Sangre del Maule.

Los resultados permiten concluir que para responder de manera eficiente y efectiva a las necesidades de sangre inmediatamente después de un desastre es de importancia clave tener un sistema centralizado de gestión que facilite el abastecimiento y el suministro de sangre y contar con personal voluntario competente en salud que esté dispuesto a acudir con celeridad durante estos eventos. Asimismo, se ha observado que durante dichas emergencias se produce un cambio en el perfil de quienes donan sangre. En Chile, por ejemplo, durante las dos semanas siguientes al terremoto la razón hombre/mujer en los donantes se invirtió, con $61,1 \%$ de participación por parte de las mujeres, quienes en la semana previa al evento representaban a solo $37 \%$.

Palabras clave Bancos de sangre; planificación de desastres; terremotos; donadores de sangre; atención de emergencias; Chile.

En la primera década de este siglo el mundo ha enfrentado diversas catástrofes, algunas naturales como inundaciones, terremotos y tsunamis, y otras provocadas por el hombre como guerras civiles y ataques terroristas. Año tras año, estos eventos provocan la pérdida de cientos de miles de vidas humanas,

\footnotetext{
Universidad de Talca, Departamento de Bioquímica Clínica e Inmunohematología, Talca, Chile. La correspondencia se debe dirigir a Marcela Vásquez, mvasquro@utalca.cl

2 Centro Productivo Regional de Sangre del Maule, Talca, Chile.
}

nuevos patrones de enfermedades, sobre todo infecciosas, y multimillonarios costos materiales. También dejan a su paso cantidades masivas de heridos de distinta gravedad, cuya atención suele representar una carga que supera la capacidad de respuesta de los servicios de salud, más aún si parte de esos servicios han sido afectados directamente. Los bancos de sangre, un componente sanitario esencial en casos de desastre, llegado el momento se encuentran con que no pueden satisfacer las nuevas y urgentes necesidades de sangre y componentes que se les presentan (1).
Ha habido sin embargo situaciones que resultaron trascendentales para el aprendizaje de la respuesta que puede dar el sistema sanitario frente a la ocurrencia de un desastre, en particular desde los bancos de sangre. Un ejemplo es el ataque terrorista del 11 de septiembre de 2001 en Nueva York. En 2003 la Asociación Americana de Bancos de Sangre (AABS) publicó un manual operativo para casos de desastres, definidos como "desastres de la naturaleza o acto terrorista" que: i) repentinamente genera una demanda de sangre más alta que la habitual, o ii) temporalmente restringe o eli- 
mina la capacidad del servicio de recolectar, analizar, procesar y distribuir la sangre, o iii) genera una afluencia masiva de donantes que produce dificultades en el sistema de recolección (2).

Para resguardar el abastecimiento de sangre en situaciones de desastre, es importante saber que su manejo sistémico incluye tres etapas: i) prevención o preparación, que consiste en contar con un plan de contingencia permanentemente actualizado, ii) respuesta, que corresponde a la definición de las acciones que se deben ejecutar una vez ocurrido el desastre; y iii) rehabilitación o recuperación, que se refiere a la etapa post emergencia, cuando es necesario recuperar, en el menor tiempo posible, el funcionamiento normal del servicio y documentar las experiencias positivas y negativas que ayuden a enfrentar de mejor forma potenciales eventos futuros (3).

En la madrugada del 27 de febrero de 2010, un terremoto de magnitud 8,8 en la escala de Ritcher sacudió el centro-sur de Chile, dejando un saldo de 521 muertos y 600 heridos, aparte de miles de hogares afectados directamente y cuantiosos daños materiales, sobre todo en infraestructura $(4,5)$. En tal sentido, los daños producidos en hospitales de la región dejaron a los servicios de salud, y entre ellos el abastecimiento y suministro de sangre, ante el desafío de superar de inmediato las dificultades y restablecerse para brindar atención a las víctimas de la catástrofe.

El presente trabajo se propone precisamente caracterizar las acciones llevadas a cabo por el Centro Productivo Regional de Sangre del Maule (CPRSM) para responder a las necesidades de sangre tras el terremoto ocurrido en 2010, comparando su gestión de recolección y suministro de sangre y componentes con las realizadas durante otras situaciones de desastre. El objetivo fue proporcionar información que pueda ser útil para mejorar las estrategias y planes de respuesta durante potenciales desastres futuros.

La información fue obtenida mediante entrevistas con funcionarios del CPRSM y de informes enviados por el Centro a la Dirección del Servicio de Salud del Maule. En el caso particular de las donaciones de sangre efectuadas entre el 22 de febrero y el 12 de marzo del 2010, los datos fueron extraídos del sistema informático del Centro (programa SBS, de Biomerieux).

\section{El sistema de gestión de sangre en la Región del Maule y en el país}

En 2007 el Ministerio de Salud de Chile creó la Comisión Nacional de Sangre y Tejidos con los objetivos de mejorar la práctica de la medicina transfusional, minimizar las pérdidas y reducir los costos de producción de hemocomponentes. La Comisión definió tres líneas de trabajo dirigidas a: i) impulsar la donación altruista de sangre, la cual en 2007 alcanzaba apenas $9 \%$, muy por debajo de la meta de $50 \%$ planteada por la Organización Mundial de la Salud para 2010, ii) racionalizar la práctica transfusional, y iii) centralizar los procesos productivos con la idea de reducir a un máximo de cuatro el número de establecimientos productores de sangre y componentes para transfusiones con cobertura supraregional.

Tal reorganización resultó en un total de tres centros regionales -en Concepción, Santiago y Valparaíso- encargados de atender las necesidades de sangre de las unidades de medicina transfusional (UMT) de hospitales y centros de salud en su área correspondiente $(6,7)$. De forma transitoria, en algunas regiones del país la gestión de sangre y componentes se ha centralizado por servicio de salud. Fue así como en abril de 2008 inició su funcionamiento el Centro Productivo Regional de Sangre del Maule, el cual en agosto de 2009 ya había conseguido centralizar el abastecimiento de sangre a nivel regional.

La Región del Maule tiene una superficie de $30296 \mathrm{~km}^{2}$ y una población total de 908097 habitantes distribuidos en cuatro provincias: Talca (352 966), Linares (252 900), Curicó (244 053) y Cauquenes (57 088). La red hospitalaria pública de la región está constituida por 13 hospitales y su sistema de sangre, como se mencionó, está centralizado en el CPRSM.

El Centro tiene un promedio mensual de 800 donantes aceptados, cuya captación y atención la realiza, además del Centro, el personal de las UMT de cinco centros adicionales correspondientes a hospitales de Curicó, Constitución, Linares, Parral y Cauquenes, desde donde provienen $33,0 \% ; 3,6 \% ; 12,0 \% ; 4,0 \%$ y $8,0 \%$ de las donaciones mensuales, respectivamente. El Centro funcionaba en la ciudad de Talca, capital de la región, y al 26 de febrero de 2010 contaba con una dependencia de $380 \mathrm{~m}^{2}$ ubicada dentro del recinto del Hospital Regional de Talca.

\section{El terremoto de 2010}

Dada su cercanía respecto al epicentro, la Región del Maule fue una de las zonas más afectadas por el sismo. De sus 13 hospitales públicos, los de Cauquenes, Curicó, Parral y Talca quedaron inhabitables, dificultando la captación de donantes de sangre. Asimismo, inmediatamente después del sismo se interrumpieron los servicios de agua, electricidad, telecomunicaciones y transporte, restringiendo de manera temporal el CPRSM para colectar, analizar, procesar y distribuir la sangre y componentes, a lo cual se sumó el hecho de que en los días siguientes se produjo una afluencia masiva de donantes, algo que concuerda con dos de los criterios establecidos por la Asociación Americana de Bancos de Sangre para clasificar una situación como desastre.

\section{Abastecimiento de sangre post desastre}

La estrategia de respuesta a un desastre siempre depende, entre otros factores, de las características particulares del evento. En el caso del terremoto del 27 de febrero, dado al relativamente bajo número de heridos, la demanda de transfusiones en la UMT del Hospital Regional de Talca, así como en las restantes de la región, en los primeros días fue mínima y pudo ser cubierta con las reservas disponibles en las UMT respectivas. Esto obedeció a que el día previo al evento cada una disponía de reservas de sangre para un funcionamiento de siete días, haciendo innecesario el reabastecimiento inmediato.

Desafíos y acciones durante las primeras 72 horas. Dentro de las primeras 72 horas posteriores al terremoto, en el ámbito del CPRSM se adoptaron una serie de medidas dirigidas a asegurar la provisión adecuada de sangre y componentes, entre las cuales destacan las siguientes (cuadro 1):

- Aun cuando la planta física del Centro quedó inhabilitada, las acciones iniciadas aproximadamente a 3 horas de ocurrida la catástrofe permitieron rescatar los productos sanguíneos almacenados y etiquetados que, incluso cuando estaban en contenedores $\mathrm{CO}^{-}$ nectados a la red eléctrica de emergencia, corrían el riesgo de no poder 
CUADRO 1. Medidas adoptadas por el Centro Productivo Regional de Sangre del Maule (CPRSM) dentro de las 72 horas siguientes al terremoto del 27 de febrero de 2010, Chile

\begin{tabular}{|c|c|c|c|}
\hline $\begin{array}{l}\text { Tiempo desde } \\
\text { el desastre } \\
\text { (horas) }\end{array}$ & Objetivo & Medida & Resultado \\
\hline \multirow[t]{2}{*}{24} & $\begin{array}{l}\text { Resguardar la reserva de hemo- } \\
\text { componentes almacenados en el } \\
\text { CPRSM }\end{array}$ & $\begin{array}{l}\text { - Inspeccionar el edificio y el equipamiento } \\
\text { - Constatar el buen funcionamiento del sistema } \\
\text { eléctrico de emergencia }\end{array}$ & $\begin{array}{l}\text { - No hubo daños aparentes en los equipos pero } \\
\text { sí en la planta física } \\
\text { - El sistema eléctrico funcionaba }\end{array}$ \\
\hline & $\begin{array}{l}\text { Velar por el suministro de hemo- } \\
\text { componentes para los heridos }\end{array}$ & $\begin{array}{l}\text { - Rescatar algunos hemocomponentes } \\
\text { etiquetados, resguardando sus condiciones de } \\
\text { almacenamiento } \\
\text { - Coordinar las comunicaciones desde y hacia las } \\
\text { unidades de medicina transfusional }\end{array}$ & $\begin{array}{l}\text { - Se trasladaron hemocomponentes a } \\
\text { dependencias más seguras } \\
\text { - Se utilizaron refrigeradores domésticos para } \\
\text { mantener la cadena de frio, mientras se } \\
\text { rescataban los del CPRSM } \\
\text { - Se fidelizó la información sobre el lugar de } \\
\text { funcionamiento de emergencia del CPRSM y las } \\
\text { demandas de sangre de los hospitales vecinos }\end{array}$ \\
\hline 48 & $\begin{array}{l}\text { Evaluar factibilidad para reiniciar } \\
\text { funcionamiento del Centro, } \\
\text { cumpliendo estándares de calidad } \\
\text { Incrementar la seguridad } \\
\text { microbiológica de los } \\
\text { hemocomponentes }\end{array}$ & $\begin{array}{l}\text { - Encontrar y preparar un nuevo espacio físico } \\
\text { para instalar áreas básicas del CPRSM } \\
\text { - Evaluar la disponibilidad de recursos humanos y } \\
\text { la funcionalidad de los equipos } \\
\text { - Eliminar las unidades con estudios de tamizaje } \\
\text { microbiológico pendientes }\end{array}$ & $\begin{array}{l}\text { - Se programó todo lo necesario para reiniciar las } \\
\text { actividades al día siguiente } \\
\text { - Se contactó personal voluntario competente } \\
\text { - Se dieron de baja las unidades riesgosas }\end{array}$ \\
\hline 72 & $\begin{array}{l}\text { Resguardar abastecimiento } \\
\text { de sangre para la región, evitando } \\
\text { la sobre-colección }\end{array}$ & $\begin{array}{l}\text { - Reiniciar el funcionamiento de las actividades } \\
\text { propias del Centro } \\
\text { - Garantizar un abastecimiento centralizado para } \\
\text { los próximos días }\end{array}$ & $\begin{array}{l}\text { - Se reiniciaron la atención de donantes y la } \\
\text { producción de hemocomponentes } \\
\text { - Se centralizó la recolección de sangre } \\
\text { únicamente en el CPRSM }\end{array}$ \\
\hline
\end{tabular}

Fuente: elaborado por los autores.

ser recuperados con posterioridad si el daño del edificio se acrecentaba a raíz de las réplicas.

- A poco más de 48 horas de ocurrido el terremoto, ya se estaba en condiciones de reiniciar la atención de los donantes de sangre para resguardar el abastecimiento de la región. Con este fin se informó a la comunidad acerca de la necesidad de donantes voluntarios, indicándose lugar y horario de atención, echando mano a la única radioemisora local que estaba transmitiendo. El hecho de que este fuera el único medio escuchado por gran parte de la población regional generó una afluencia masiva de donantes de sangre, entre el 1 y el 12 de marzo, que permitió colectar 561 donaciones efectivas. La mayoría se concentró durante la primera semana de ocurrido el sismo, con un incremento de 2,5 veces en el número de donaciones respecto de la semana previa (441 vs. 175), en tanto en la segunda semana el total de donaciones fue inferior en relación a la misma semana (120 vs. 175) (figura 1).

- Las decisiones tomadas durante la emergencia contemplaron el cumplimiento de los estándares de calidad necesarios para resguardar la seguridad en el proceso de producción de los

FIGURA 1. Distribución por sexo y edad promedio de los donantes de sangre atendidos en el Centro Productivo Regional de Sangre del Maule una semana antes y dos semanas después del terremoto del 27 de febrero de 2010, Chile

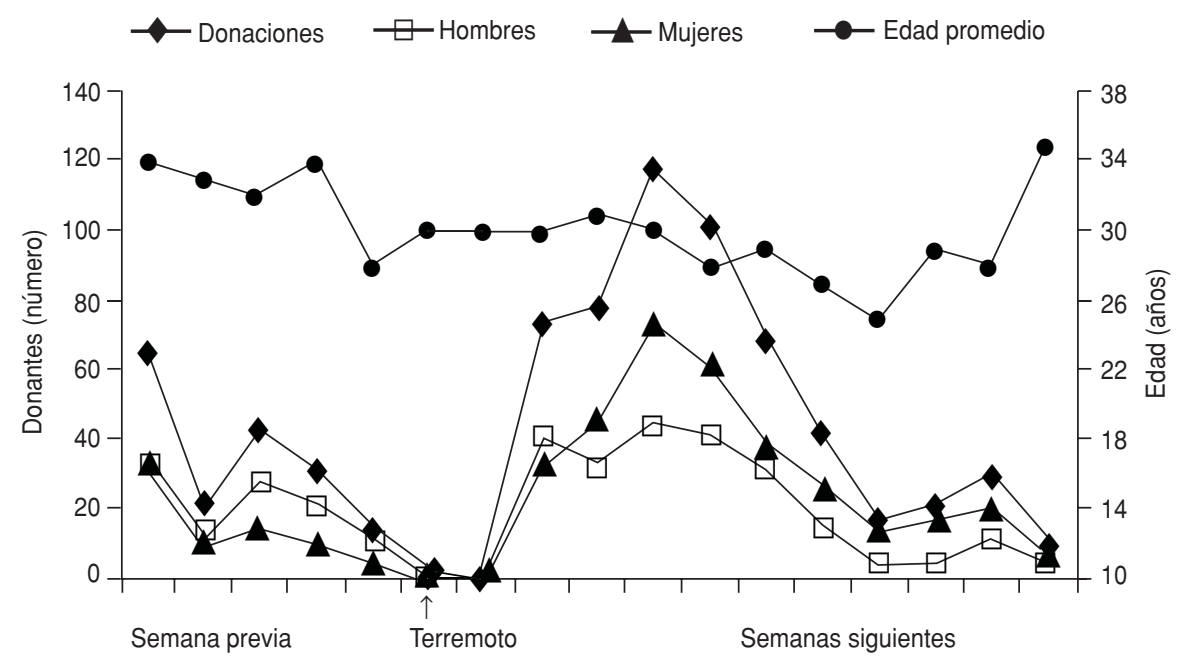

Fuente: elaborado por los autores.

hemocomponentes, como por ejemplo contar con personal voluntario competente conformado, entre otros, por docentes y alumnos de último nivel de la Escuela de Tecnología Médica de la Universidad de Talca. Esta medida permitió brindar atención oportuna y expedita a los más de 90 donantes que en promedio concurrieron cada uno de los cuatro primeros días desde que se reinició la atención, cifra muy superior al promedio de donantes que se atienden en condiciones normales.

- Se destaca también la decisión de eliminar las unidades que permanecían en cuarentena —que habían dado positivo en estudios microbiológicos y debían ser reevaluadas- para evitar que, 
en la emergencia, pudiesen ser consideradas aptas para ser transfundidas.

- A las pocas horas de ocurrido el desastre, y en la medida que se restablecían las comunicaciones, ya se estaba recabando información sobre la demanda de sangre de las distintas UMT, pues existía conocimiento de que la determinación de las necesidades de sangre inmediatas y a corto plazo es uno de los puntos críticos de la etapa de respuesta $(2,8,9)$. Además, para el cuidado de las reservas de sangre se contó con el apoyo de los servicios clínicos, como por ejemplo las unidades de urgencia, en donde mediante escalas de triage se racionalizaron las indicaciones de transfusiones sanguíneas (10).

\section{Caracterización de las donaciones de sangre post desastre}

Las experiencias en distintos desastres ocurridos en el mundo han mostrado que los donantes en estos casos tienen perfiles diferentes respecto de quienes donan sangre en situaciones normales. En Chile, durante las dos semanas siguientes al terremoto de 2010 la razón hombre/mujer en los donantes se invirtió, con $61,1 \%$ de participación por parte de las mujeres, quienes en la semana previa al evento representaban a solo $37 \%$ de los donantes (figura 1).

Asimismo, se encontró que 97,6\% de quienes concurrieron a dar sangre en las dos semanas siguientes al terremoto fueron voluntarios, mientras que en 2009 este tipo de donantes representó un 19\%. Más todavía, de los donantes voluntarios post evento, $81,4 \%$ lo hacían por primera vez, mientras que en la semana previa este tipo de voluntarios representó 58,8\% del total (figura 2). Del total de personas que donaron sangre los primeros 12 días post catástrofe, solo dos $(0,36 \%)$ dieron positivo a sífilis, mientras que en 2009 los donantes que dieron positivo a esa enfermedad alcanzaron el $0,82 \%$.

\section{DISCUSIÓN}

Dado que tras la ocurrencia de un desastre la comunidad local se muestra mucho más dispuesta a realizar acciones altruistas, el abastecimiento de sangre en general no representa un problema complejo (11-13), como lo prueba el incremento de 2,5 veces en las donaciones voluntarias durante la semana siguiente

FIGURA 2. Donantes voluntarios de primera vez y repetidos que donaron sangre en el Centro Productivo Regional de Sangre del Maule una semana antes y dos semanas después del terremoto del 27 de febrero de 2010, Chile

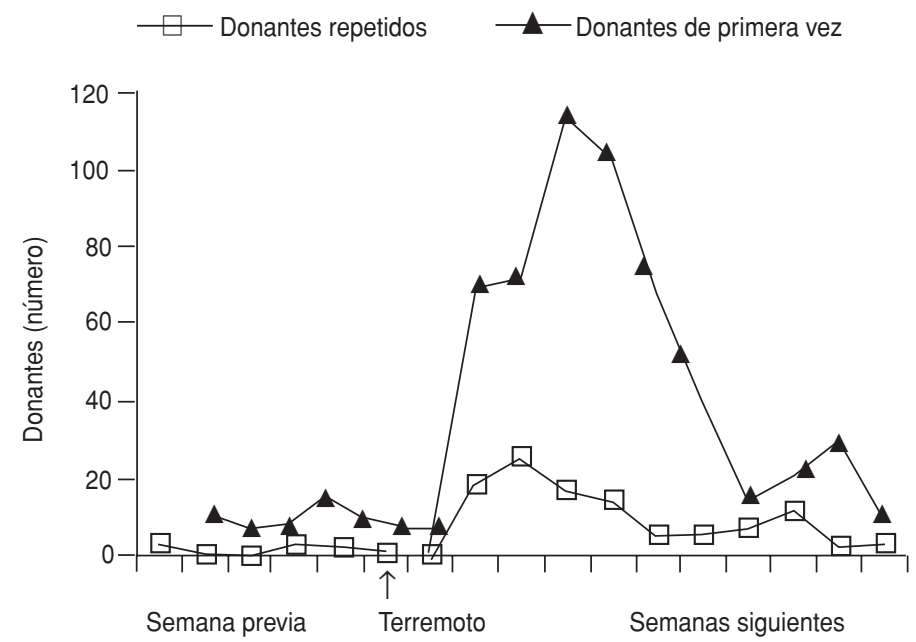

Fuente: elaborado por los autores.

a las catástrofes de Nueva York en 2001, Sri Lanka en 2004 y Chile en 2010, y de 6,9 veces en Bam (Irán) en 2003 (1, 14, 15).

Lo notificado por la literatura así como la experiencia en la región chilena del Maule demuestran que los desastres naturales no generan una gran demanda inmediata de sangre. En un informe sobre la sangre usada con posterioridad a distintos desastres ocurridos en Estados Unidos entre 1976 y 2001, Paul Schmidt constató que en todos los casos las necesidades fueron cubiertas con la sangre disponible y que la sangre aportada por donantes tras los llamados a la comunidad finalmente no fue necesaria $(11,16)$.

Las cantidades de sangre colectadas inmediatamente después de un desastre deben ser las suficientes para satisfacer las necesidades transfusionales de la emergencia, sin caer en una colección excesiva de unidades que más tarde seguramente serán eliminadas. Un ejemplo tuvo lugar en Nueva York después del ataque del 11 de septiembre cuando, debido a una falta de coordinación entre la Cruz Roja y el Departamento de Salud y Recursos Humanos, los volúmenes excesivos de sangre y componentes provocaron una crisis de mercado en que la oferta sobrepasó a la demanda $(11,13$, 17). De acuerdo a la experiencia del Maule, el contar con un modelo centralizado, además de sus ventajas económicas y de calidad, fue instrumental al momento de enfrentar la emergencia porque las decisiones respecto al abastecimiento de sangre se concentraron en el CPRSM, evitando descoordinaciones que podrían haber repercutido negativamente en la etapa de respuesta (18).

Sri Lanka, un país que ha sufrido diferentes tipos de catástrofes, es un ejemplo de cómo hay que manejar el sistema de sangre para estar preparados al momento de una emergencia masiva (1). Su gestión de sangre y componentes es coordinada por el Servicio Nacional de Transfusiones de Sangre (NBTS, por sus siglas en inglés) y su experiencia puede resumirse en cinco recomendaciones, de las cuales tres fueron aplicadas exitosamente en el terremoto de Chile de 2010: i) asegurar que todos los servicios de transfusión estén permanentemente preparados para desastres, manteniendo reservas de sangre y componentes suficientes para siete días; ii) controlar que la colección de sangre no sea insuficiente ni excesiva, sino que se ajuste a la demanda, y iii) hacer llegar a la comunidad de donantes y al público en general mensajes claros y coherentes acerca de las necesidades de sangre.

La experiencia en el Maule concuerda con lo observado en la literatura en el sentido de que inmediatamente después de un desastre hay un cambio en el perfil de los donantes de sangre voluntarios. Por ejemplo, tanto en Chile 2010 como en Nueva York 2001 y Sichuan (China) 2008 se registró una inversión en la razón hombre/mujer de donantes, con una participación mucho más alta de mujeres $(14,19)$. Se observa asimismo que los desastres ejercen una fuerte motivación sobre la población para donar sangre por 
primera vez de forma voluntaria, evidenciado en incrementos de este tipo de donantes de 22,6\% en el Maule; 26\% en Nueva York y 7,9\% en Sichuan $(14,19)$. Este cambio en el tipo de donantes podría elevar el riesgo absoluto de infecciones virales transmitidas por transfusión, puesto que los marcadores infecciosos de esta población suelen ser mayores que en los donantes repetidos. Sin embargo, incluso cuando este fenómeno fue notificado en relación con las donaciones de sangre tras el ataque terrorista en Nueva York, no sucedió lo mismo en los casos de Marmara (Turquía) 1999, Sichuan 2008 y Chile 2010, donde los marcadores infecciosos en la semana siguiente a los desastres no mostraron diferencias significativas con respecto al resto del año (19-21). En este sentido, el aumento de donantes de primera vez representa una oportunidad para elevar la cantidad de donantes repetidos, por lo cual cada centro de sangre debería generar un plan de reclutamiento que permita aprovechar la mayor afluencia de voluntarios post desastre para contactarlos en el futuro (15).

La calidad y la seguridad de la sangre colectada en situaciones de desastre se ven afectadas no solo por las características de la población donante, sino por el grado de idoneidad en los procesos de selección y atención de los donantes y de fraccionamiento, seguridad serológica y almacenamiento de los hemocomponentes. En relación con esto se ha observado que, usando como excusa la emergencia, no se mantienen los estándares de calidad de los procesos y se tiende a priorizar la cantidad sobre la calidad de la sangre colectada $(9,22)$. Una forma de prevenir estas situaciones es contar con personal voluntario competente, algo que en Chile 2010 se logró gracias al apoyo de docentes y alumnos universitarios de carreras afines.

\section{Conclusiones}

Los informes publicados acerca de la gestión de la sangre durante desastres, sumados a la experiencia de Chile después del terremoto de 2010, permiten concluir que: i) aun en eventos de distintos tipos y gravedad en términos de impacto en las personas no se hallan diferencias importantes en cuanto al manejo del abastecimiento y suministro de sangre, ni a las características de las donaciones; ii) contar durante la emergencia con un voluntariado competente (p. ej. alumnos y docentes universitarios de carreras afines) ayuda a resguardar la calidad de los distintos procesos asociados al banco de sangre; iii) al momento de enfrentar una situación de catástrofe, el modelo centralizado de banco de sangre facilita la gestión de respuesta, ya que permite concentrar en una sola entidad la toma de decisiones sobre el abastecimiento y suministro, previniendo de este modo descoordinaciones que normalmente traen aparejadas duplicación del trabajo, desbalances en el abastecimiento de sangre y otros problemas que restan efectividad a los esfuerzos desplegados.

\section{REFERENCIAS}

1. Kuruppu K. Management of blood system in disasters. Biologicals. 2010;38(1):87-90.

2. American Association of Blood Banks. Disaster Operations Handbook-Hospital Supplement. Disponible en: http://www.aabb.org/ programs/disasterresponse/Documents/ disastophndbkv2.pdf. Acceso el 28 de febrero de 2011.

3. Organización Panamericana de la Salud. Intervención de los laboratorios y bancos de sangre ante situaciones de desastre, 2001. Disponible en: http://www.paho.org/Spanish/ HSP/HSE/laboratorios.htm. Acceso el 24 de enero de 2011.

4. Ministerio del Interior, Chile. Fallecidos confirmados con RUT y Certificado de defunción de Registro Civil. Disponible en: http:// www.interior.gov.cl/filesapp/Lista falleci dos.pdf. Acceso el 24 de enero de 2011.

5. Ministerio del Interior, Chile. Denuncias por presuntas desgracias relacionadas con el terremoto y maremoto del 27 de febrero. Disponible en: http://www.interior.gov.cl/ filesapp/Lista_desaparecidos.pdf. Acceso el 24 de enero de 2011.

6. Ministerio de Salud, Chile. Dossier nacional sobre políticas de donación altruista de sangre. Disponible en: http://eurosocialsalud. eu/files/docs/00168.pdf. Acceso el 24 de enero de 2011.

7. Organización Panamericana de la Salud. Informe sobre los progresos realizados por la iniciativa regional para la seguridad sanguínea y plan de acción para 2006-2010. $46^{\circ}$ Consejo Directivo, CD46/16; 2005. Dis- ponible en: http:/ /www.paho.org/spanish/ gov/cd/cd46-16-s.pdf. Acceso el 24 de enero de 2011.

8. Shinar E, Yahalom V, Silverman BG. Meeting blood requirements following terrorist attacks: the Israeli experience. Curr Opin Hematol. 2006;13(6):452-6.

9. Mujeeb SA, Jaffery SH. Emergency blood transfusion service after the 2005 earthquake in Pakistan. Emerg Med J. 2007;24(1):22-4.

10. Reisner A. Triage. In: Ciottone G, ed. Disaster Medicine. Philadelphia: Mosby Elsevier; 2006. Pp. 283-90.

11. Schmidt PJ. Blood and disaster-Supply and demand. N Engl J Med. 2002;346(8):617-20.

12. Busch MP, Guiltinan A, Skettino S, Cordell R, Zeger G, Kleinman S. Safety of blood donations following a natural disaster. Transfusion. 1991;31(8):719-23.

13. Hess JR, Thomas MJG. Blood use in war and disaster: lessons from the past century. Transfusion. 2003;43(11):1622-33.

14. Glynn SA, Busch MP, Schreiber GB, Murphy EL, Wright DJ, Tu Y, et al. Effect of a national disaster on blood supply and safety: the September 11 experience. JAMA. 2003;289 (17):2246-53.

15. Abolghasemi $\mathrm{H}$, Radfar $\mathrm{MH}$, Tabatabaee M, Hosseini-Divkolayee NS, Burkle FM Jr. Revisiting blood transfusion preparedness: Experience from the Bam Earthquake. Prehosp Disaster Med. 2008;23(5):391-4.

16. Linden JV, Davey RJ, Burch JW. The September 11, 2001 disaster and the New York blood supply. Transfusion. 2002;42(10):1385-7.
17. Klein HG. Earthquake in America. Transfusion. 2001;41(19):1179-80.

18. Organización Panamericana de la Salud. Guía para la estimación de costos de la regionalización de los bancos de sangre. Disponible en: http://www.paho.org/Spanish/AD/ THS/EV/blood_guiaCostos.pdf. Acceso el 12 de noviembre de 2010.

19. Liu J, Huang Y, Wang J, Bi X, Li J, Lu Y, et al. Impact of the May 12, 2008, earthquake on blood donations across five Chinese blood center. Transfusion. 2010;50(9):1972-9.

20. Sönmezoglu $M$, Kocak $N$, Oncul $O$ Ozbayburtlu S, Hepgul Z, Kosan E, et al. Effects of a major earthquake on blood donor types and infectious diseases marker rates. Transfus Med. 2005;15(2):93-7.

21. Dodd RY, Orton SL, Notari EP 4th, Stramer SL. Viral marker rates among blood donors before and after the terrorist attacks on the United States on September 11, 2001. Transfusion. 2002;42(9):1240-1.

22. De Ville de Goyet C. Blood: the ultimate donation in emergencies. Prehosp Disaster Med. 2008;23(5):395-6.

Manuscrito recibido el 1 de noviembre de 2010. Aceptado para publicación, tras revisión, el 30 de diciembre de 2010. 
ABSTRACT A review of the management of blood supply and its administration during disasters was conducted based on the experience of several events that occurred primarily from

Blood supply during disasters: $2000-2010$, particularly the earthquake that measured 8.8 on the Richter scale that
the experience of the experience of Chile in 2010 information that could be useful in improving response plans and strategies during potential future disasters.

The descriptive information on response procedures was obtained from interviews, internal reports, and the computer database from the Maule regional blood production center.

The results lead to the conclusion that to respond efficiently and effectively to the need for blood in the immediate wake of a disaster it is essential to have both a centralized management system that facilitates the supply and administration of blood and volunteers with competence in health that are willing to swiftly arrive during these events. A change in the profile of blood donors during such emergencies was also observed. In Chile, for example, during the two weeks after the earthquake, the ratio of male/female donors was reversed. There was $61.1 \%$ participation by women, whereas in the week before the event women accounted for only $37 \%$.

Key words Blood banks; disaster planning; earthquakes; blood donors; emergency watch; Chile. 